\title{
Communication System Using Periodic Nonlinear Fourier Transform Based on Riemann-Hilbert Problem
}

\author{
M. Kamalian ${ }^{(1)}$, D. Shepelsky (2), A. Vasylchenkova(1), J. E. Prilepsky(1), S. K. Turitsyn ${ }^{(1)}$ \\ (1) Aston Institute of Photonic Technologies, Aston University, B4 7ET, Birmingham, UK. \\ kamaliam@aston.ac.uk \\ (2) B. Verkin Institute for Low Temperature Physics and Engineering, 61103, Kharkov, Ukraine.
}

\begin{abstract}
In a communication system based on periodic nonlinear Fourier transform, we apply the associated Riemann-Hilbert problem to modulate the nonlinear spectrum of the signal and study the performance and achievable mutual information.
\end{abstract}

\section{Introduction}

Nonlinear Fourier transform (NFT) has been studied and experimentally demonstrated recently, as a promising tool to overcome Kerr nonlinearityinduced distortions ${ }^{1,2}$. Aside from conventional NFT-based setups, using periodic NFT (PNFT) can be beneficial due to reduction of processing window and computational complexity, and control over the time duration of the signal ${ }^{3}$. However, one of the major hurdles in the PNFT implementation is the lack of a computationallyefficient method to perform the inverse transformation stage. There have been proposed some alternatives to the exact inverse $\mathrm{PNFT}^{3-5}$ : an approximation of the inverse transformation can be carried out, or some analytically known signals with limited freedom in choosing the parameters can be used ${ }^{4,5}$. While the former suffers from the inaccuracy of the results and sensitivity to system characteristics, the latter only provides onedimensional constellations with a large minimum distance leading to a high bit error rate (BER). The nonlinear spectrum (NS) of a periodic signal consists of a discrete (main) and auxiliary spectrum. Both spectra can be modulated to carry data down the fibre, but in our work, to simplify the calculations, we use only the discrete spectrum and consider a single QAM symbol per signal. According to the system properties and desired time duration of the signal, a randomly drawn QAM symbol is rescaled and considered as an eigenvalue (a member of the discrete spectrum). Then, a signal with a modulated main spectrum is constructed and sent to the fibre. At the receiver, the NS of the signal is calculated, and the data is retrieved. Calculating the NS of a periodic signal, i.e. the direct PNFT, is similar to the NFT for vanishing boundary: it can be done by using a fast algorithm in which the Zakharov-Shabat system of differential equations (ZSS) ${ }^{6}$

$$
\boldsymbol{\Phi}_{t}=\left[\begin{array}{cc}
-i \lambda & q(t, z) \\
-q(t, z)^{*} & i \lambda
\end{array}\right] \boldsymbol{\Phi},
$$

associated with the normalised nonlinear Schrödinger equation (NLSE) written here explicitly for anomalous dispersion,

$$
i q_{z}+q_{t t}+2|q|^{2} q=0
$$

is solved using polynomial arithmetic.

The inverse transformation, on the other hand, has significant differences compared to its vanishing signal counterpart and should be addressed in more details. In the following, we explain a computationally feasible framework to construct a periodic signal from its NS.

\section{Inverse transformation for periodic signals}

Constructing a signal from its NS can be rendered using Riemann theta function, a multidimensional Fourier series ${ }^{7}$. Although there have been attempts to reduce the computational complexity of calculating this function in recent years 8,9 , it is still not applicable for a real-time application such as fibre-optic communication. Use of Riemann theta function can be avoided by means of formulating the inverse transformation stage as a Riemann-Hilbert (RH) problem ${ }^{10}$. Within such an approach, starting from some cuts in the complex plane, one needs to find a (matrix) function analytic everywhere in the complex plane except on the cuts, having a prescribed jump across the cut and a particular (related to the NLSE) prescribed behaviour at infinity. Having the $\mathrm{RH}$ problem solved, the solutions of the associated ZSS as well as the solution to the NLSE can be derived ${ }^{10}$. The desired properties of the latter (i.e. periodicity) can be ensured by an appropriate choice of cuts and jumps. 


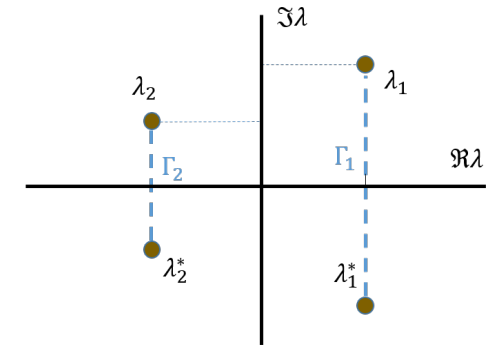

Fig. 1: The nonlinear spectrum of a periodic solution with cuts $\Gamma_{1}$ and $\Gamma_{2}$. Circles represent the discrete spectrum and dashed lines denote the cuts.

\section{PNFT-based communication system}

In our work, for the first time, we apply the $\mathrm{RH}$ problem method in NFT-based system. Since it is sufficient to have only two degrees of freedom in order to represent a QAM symbol in the discrete spectrum, we restrict our calculations to the simple case where we have only two cuts in the NS, Fig. 1. According to the drawn QAM symbol and by considering the required time duration of the signal, two points in the complex plane, $\lambda_{1}$ and $\lambda_{2}$, are chosen, generating two cuts $\Gamma_{1}$ and $\Gamma_{2}$, see Fig. 1. Then the corresponding jumps in the form of matrices below are designed ${ }^{10}$ :

$$
\begin{aligned}
& \mathbf{J}_{1}=\left[\begin{array}{cc}
0 & i e^{-i \phi_{1}} \\
i e^{i \phi_{1}} & 0
\end{array}\right], \\
& \mathbf{J}_{2}=\left[\begin{array}{cc}
0 & -i e^{i\left(C^{f} t+C^{g} z+\phi_{2}\right)} \\
i e^{i\left(C^{f} t+C^{g} z+\phi_{2}\right)} & 0
\end{array}\right],
\end{aligned}
$$

where $C^{f, g}$ are frequencies defined from $\lambda_{1,2}$, and $\phi_{1,2}$ are arbitrary phases. To solve the RH problem means to find a matrix, $\mathrm{M}$, with the following properties $^{10}$

$$
\begin{gathered}
\mathbf{M}^{-}(t, z, \lambda)=\mathbf{M}^{+}(t, z, \lambda) \mathbf{J}_{j}, \text { for } \lambda \in \Gamma_{j}, j=1,2, \\
\mathbf{M}(t, z, \lambda \rightarrow \infty) \rightarrow \mathbf{I} .
\end{gathered}
$$

Calculating $\mathbf{M}_{1}(t, z)$ from the asymptotic condition,

$$
\mathbf{M} \sim \mathbf{I}+\mathbf{M}_{1} \lambda^{-1}+O\left(\lambda^{-2}\right) \text { as } \lambda \rightarrow \infty,
$$

allows us to construct the solution as

$$
q(t, z)=2 i\left(\mathbf{M}_{1}\right)_{1,2}(t, z) .
$$

This signal is then cyclically extended for the duration that equals the dispersion-induced signal broadening at the transmission distance (see Fig. 2). Given the signal duration, periodicity determines the real part of $\lambda_{1,2}$. Thus, we have two real parameters, - the imaginary parts of the eigenvalues, - on which we map the QAM symbol. Then the QAM constellation is formed by $\Im \lambda_{1}$

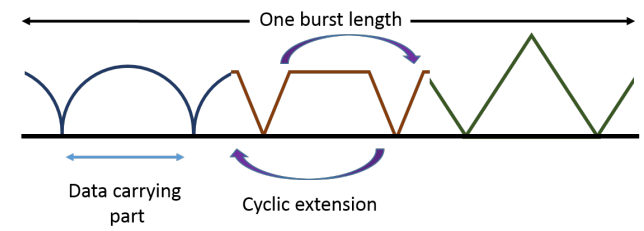

Fig. 2: Cyclic extension for a periodic signal to overcome the intersymbol interference caused by signal broadening.

and $\Im \lambda_{2}$, see Fig. 1 and smaller panes in Fig. 3.

Although here we only use two eigenvalues in the main spectrum and consider one QAM symbol per signal, our proposed procedure of constructing signal can be generalised to the case with several symbols per signal. In such a case, several cuts ending with some eigenvalues are formed in such a way that: 1) we form a periodic solution, and 2) we gain many parameters apt to the modulation of random data. However, this is left for future work.

\section{Simulation results}

In a fibre link consisting of several spans of length $L=80 \mathrm{~km}$ with parameters $\beta_{2}=-20 \mathrm{ps}^{2} / \mathrm{km}$ and $\gamma=1.3 / \mathrm{W} / \mathrm{km}$, we transmit a sequence of $2^{10}$ bursts of signals with $10 \times M$ symbols in each burst, where $M$ is the size of the constellation. We assume a constant signal power provided by a second order Raman amplification scheme. To solve the $\mathrm{RH}$ problem numerically, we use a Mathematica package RHPackage ${ }^{11}$, where the number of samples and parameters are chosen to have a balance between the accuracy and computational complexity. As mentioned before, the exact inverse PNFT makes it possible to control signal power and time duration. Changing the signal power takes place through our changing the imaginary part of eigenvalues, while by altering the real eigenvalues' part we can tune the signal bandwidth. Using these properties, we calculated the $Q^{2}$-factor at the receiver, Fig. 3, using the error vector magnitude (EVM). In this figure, the $Q^{2}$ factor against signal power for two constellation sizes $M=4$ and $M=16$, and their respected receiver constellation at the optimum power, are shown. The transmission distance is $z=880 \mathrm{~km}$, and the symbol rate is $0.8 \mathrm{Gsym} / \mathrm{s}$. The length of the cyclic prefix is set to the dispersion-induced signal memory at $1000 \mathrm{~km}$. Using the NFT, the communication takes place through a linearised channel in which the NS undergoes a linear distortion as opposed to the nonlinear evolution of signal. An important characteristic of such a medium is the noise distribution which we depict in Fig. 4 as a 2-dimensional histogram of the re- 


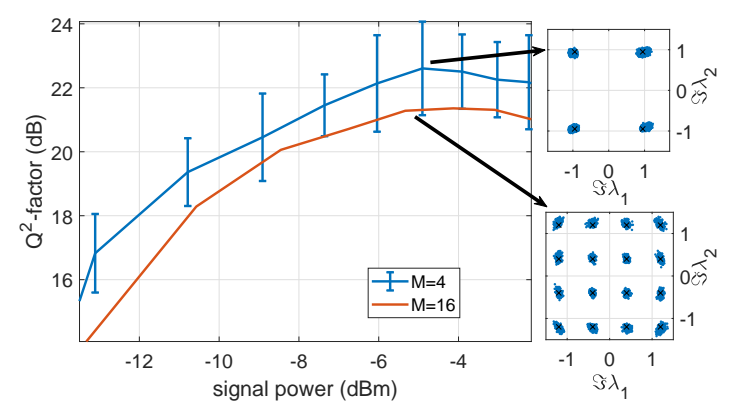

Fig. 3: $\mathrm{Q}^{2}$-factor against signal power with constellation size $M=4,16$ for a $0.8 \mathrm{Gsym} / \mathrm{s}$ transmission up to $z=880 \mathrm{~km}$.

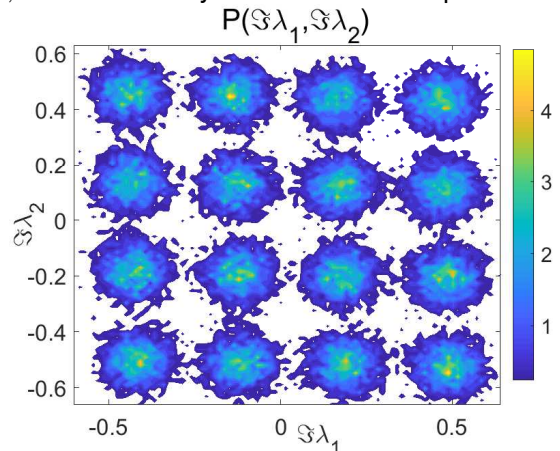

Fig. 4: Empirical joint probability density function of $\Re \lambda_{1}$ and $\Re \lambda_{1}$ for constellation size $M=16$ at distance $1040 \mathrm{~km}$ with signal power $P=-12 \mathrm{dBm}$.

ceived points. We observed that there is an almost Gaussian probability density describing the stochastic characteristics of our channel. Then there is a dependence of the variance of the noise on the location of symbols, especially at high powers (not shown here). The larger is the power, the larger is the imaginary part of our eigenvalues. Hence we expect to observe a stronger noise (greater variance) at the top right side of the constellation.

The important metric of the information system's performance is the mutual information (MI). In our case we calculate it between the transmitted eigenvalue, $\lambda$, and the received one, $\hat{\lambda}$. Fig. 5 demonstrates the achievable MI for our PNFT system employing the $\mathrm{RH}$ problem-based signal generation at the transmitter, measured at various link lengths. In particular, our study showed that for the simpliest PNFT system studied here we can reach over $6.5 \mathrm{bits} / \mathrm{sym}$ at $1200 \mathrm{~km}$.

\section{Conclusion}

In our study, to enhance the flexibility and performance of PNFT-based communication systems we propose to use the $\mathrm{RH}$ problem-based approach: the inverse transformation stage of the PNFT is performed by means of solving a $\mathrm{RH}$ problem, and the constructed signal is used in a fibre-optic communication system. The performance of this system is shown through the $Q^{2}$ factor against power and achievable mutual infor-

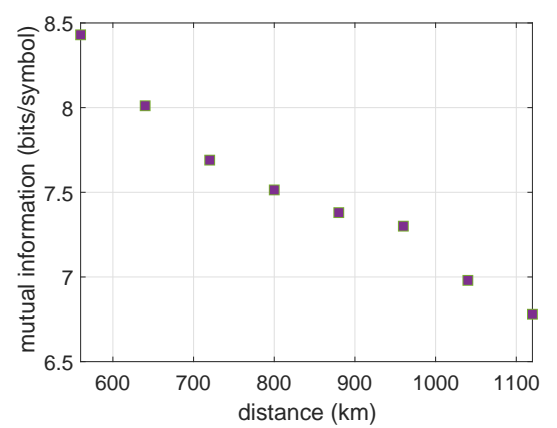

Fig. 5: Achievable mutual information at each distance for a PNFT system with one QAM symbol per signal.

mation at different distances. The proposed procedure of constructing periodic signals with arbitrary nonlinear spectrum is generalisable to render a multi-symbol per signal communication to reach higher spectral efficiency.

\section{Acknowledgements}

This work was partially supported by the Leverhulme Project RPG-2018-063.

\section{References}

[1] S. Turitsyn et al, "Nonlinear Fourier transform for optical data processing and transmission: advances and perspectives," Optica, Vol. 4, p. 307-322 (2017).

[2] S. Le, V. Aref, and H. Bülow, "Nonlinear signal multiplexing for communication beyond the Kerr nonlinearity limit," Nat. Photonics, Vol. 11, p. 570-576 (2017).

[3] M. Kamalian et al, "Periodic nonlinear Fourier transform for fiber-optic communications, Part I: theory and numerical methods, Part II: eigenvalue communication," Opt. Express, Vol. 24, p. 1835 (2016).

[4] M. Kamalian et al, "Spectral efficiency estimation in periodic nonlinear Fourier transform based communication systems," Proc. OFC, Th2A.54 (2017).

[5] M. Kamalian et al, "Optical communication based on the periodic nonlinear Fourier transform signal processing," 6th ICP, Kuching (2016).

[6] S. Wahls, and H. Poor, "Fast numerical nonlinear Fourier transforms," IEEE T. Inform. Theory, Vol. 61 (2015).

[7] E. D. Belokolos, et al Algebro-geometric Approach in the Theory of Integrable Equations, Springer, Berlin, 1994.

[8] B. Deconinck, et al. "Computing Riemann Theta Functions," Math. Comput., Vol. 73, p. 1417-1442 (2003).

[9] A. Osborne, "The random and deterministic dynamics of 'rogue waves' in unidirectional, deep-water wave trains," Mar. Struct., Vol. 14, p. 275-293 (2001).

[10] V. Kotlyarov and D. Shepelsky, "Planar unimodular Baker-Akhiezer function for the nonlinear Schrödinger equation," Ann. Math. Sci. Appl., Vol. 2 (2017).

[11] S. Olver, RHPackage for MATHEMATICA, http://www . maths. usyd.edu.au/u/olver/ projects/RHPackage.html 